\title{
Effects of perturbations on a trained shape memory micro-actuator
}

\author{
Y. Bellouard, R. Clavel and R. Gotthardt ${ }^{1}$ \\ Institut de Systèmes Robotiques, ISR, Département de Microtechnique, Ecole Polytechnique \\ Fédérale de Lausanne, 1015 Lausanne, Switzerland \\ ${ }^{1}$ Institut de Génie Atomique, IGA, Département de Physique, École Polytechnique Fédérale \\ de Lausanne, 1015 Lausanne, Switzerland
}

\begin{abstract}
The so-called Two-Way-Shape-Memory Effect (TWSME) introduced using a training process has been used to produce a reversible motion in a small actuator. It is usually noted that the TWSME induces a preferred oriented martensite which results, during the reverse transformation, in a spontaneous shape change on cooling. While heating up, the material transforms to austenite and recovers its original shape. However, if the material is mechanically deformed in martensite, in order to modify the martensite variants distribution induced by the TWSME, a two-step transformation occurs: when heating up, the material first transforms into the low-temperature shape, -i.e. the "memorized" shape induced by the training process"- and then to the high-temperature shape. This two-step transformation has been observed in a SMA micro-device. These experiments are presented in details and possible interpretations will be discussed.
\end{abstract}

\section{INTRODUCTION}

Among the solutions available for producing a reversible motion, the so-called Two-Way Shape Memory Effect (TWSME) has been one of the most studied. The basic principle is to introduce an internal stress within the material which will induce a preferred oriented martensite on cooling. Therefore, a spontaneous shape change will also appear on cooling giving its name to this effect. Among the methods to obtain a TWSME, one is to apply a specific thermo-mechanical treatment on the material. These treatments are usually called « training processes » and have been described widely in the litterature for different kinds of material (for instance $J$. Perkins and D. Hodgson in [1]). To simplify, in this article, the acronym TWSME will be restricted to the effect induced by thermo-mechanical treatments and more specifically by « training processes $"$.

In contrast with experimental studies on the subject, successfull industrial applications of the TWSME are rather limited. Several reasons can be pointed out:

- The training procedure can be a limitation for large scale production.

- It is well known that the stress generated on cooling is rather small compared to the stress generated upon heating [2]. Therefore, only applications where limited forces are required on cooling are conceivable for the TWSME technology.

However, thanks to the TWSME, the material itself can directly be used as an actuator producing a motion without «external» help like a bias spring for instance. Therefore, the TWSME is very attractive for miniaturization. In micro-engineering, it can be applied in monolithic design even with complex geometry [3].

In a previous paper [3], a micro-gripper of about $1 \mathrm{~mm}$ square has been presented. This actuator has been developed for the manipulation of small optical lenses. In this paper, new observations done on this micro-device are presented. Effects of disturbances on the initial conditions in martensite are highlighted. 


\section{EXPERIMENTAL PROCEDURE}

The micro-device is shown on figure 1 . It consists of a mobile arm and a fixed part. The mobile part and its counterpart are the gripping jaws. Upon heating, the arm's hinge bends and closes the jaws. On cooling, the arm does the reversible motion and open the jaws. This gripper is cut out from a cold-rolled sheet using a Nd-Yag SLAB laser. The material is a ternary Ni-Ti with about $6 \%$ of $\mathrm{Cu}$. The annealing was done by laser [4]. The transformation temperatures have been measured by $\mathrm{DSC}^{1}\left(\mathrm{Mf}=40^{\circ} \mathrm{C}, \mathrm{Ms}=68^{\circ} \mathrm{C}, \mathrm{As}=58^{\circ} \mathrm{C}, \mathrm{Af}=85^{\circ} \mathrm{C}\right)$.

The training process is performed by constrained temperature cycling of deformed martensite [1]. The following procedure is applied: first, a shaft is inserted between the gripping jaws. The diameter of the shaft is chosen in order to obtain a given maximum strain within the hinge (active part on figure 1 ) equal to about $4 \%$. The system is then thermally cycled between the ambiant temperature and a temperature of about $100^{\circ} \mathrm{C}$. The applied number of training cycles are set to 50 cycles. The grasping force for a given object diameter of $350 \mu \mathrm{m}$ is about $16 \mathrm{mN}$ while the force generated on cooling is about $4 \mathrm{mN}$.

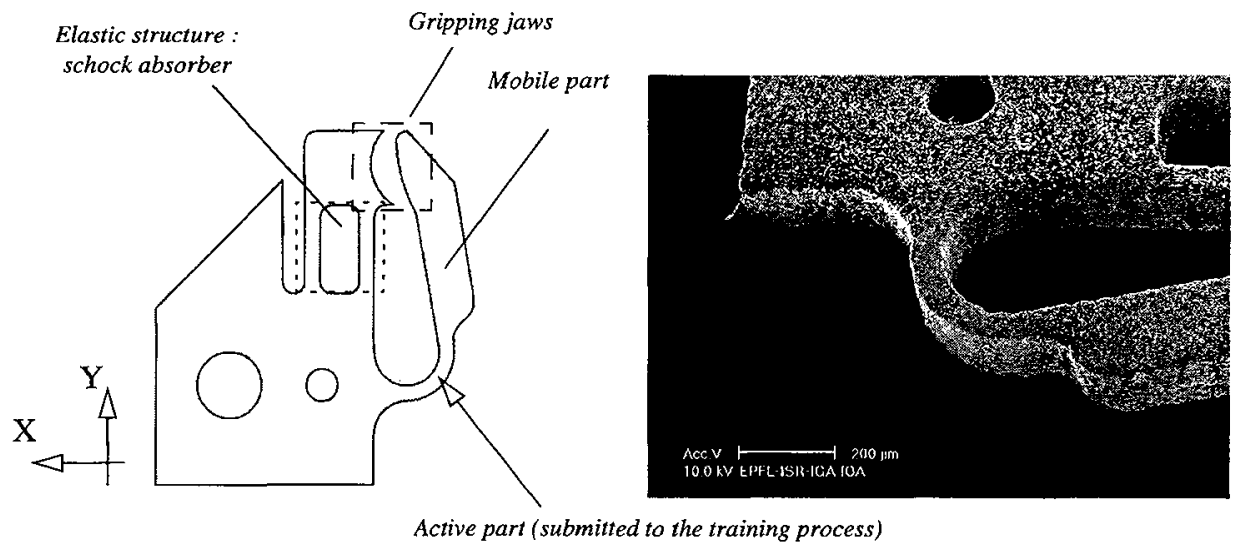

Figure 1: TWSME micro-gripper design (shown in the closed position) and a Scanning-Electron-Microscopy (SEM) view of the active zone.

The arm motion is measured using computer micro-vision [5]. This technology allows non-contact measurement within the plane. The basic principle is that a pattern simply based on the material texture is learned from the picture grabed by a CCD (Charge-Coupled Device) camera mounted on a microscope. When the gripper moves, the pattern is tracked by the vision system and a measure of the position is given each $50 \mathrm{~ms}$. The absolute accuracy mainly depends on where the pattern is chosen according known points on the device's geometry while the relative accuracy is mainly a function of the microscope magnification ratio. In measurements presented in this paper, the absolute accuracy can be estimated to $+/-10 \mu \mathrm{m}$ while the relative precision is $0.5 \mu \mathrm{m}$. Therefore the pattern matching based micro-vision technology is an accurate method for measuring relative motion within the plane.

The micro-gripper is fixed on a resistive layer deposited on a alumina substrate. This resistive layer allows to heat the device. The temperature is measured using a thermocouple with a «ball» diameter of $100 \mu \mathrm{m}$. The temperature sensor is placed directly on the resistive film and in contact with the micro-gripper. The absolute precision is estimated to about $3^{\circ} \mathrm{C}$ while the relative accuracy is $0.5^{\circ} \mathrm{C}$.

1. Differential Scanning Calorimetry 


\section{RESULTS}

The sample was submitted to successive heating and cooling cycles with a progressive increase of magnitude. The heating rate was rather slow and set to about $0.8^{\circ} \mathrm{C} / \mathrm{s}$. Progressive incomplete cycles showing the subloops in the complete cycle are shown on figure 2 . The opened position refers to the shape in the martensitic state while the closed position corresponds to the shape in the austenitic state.

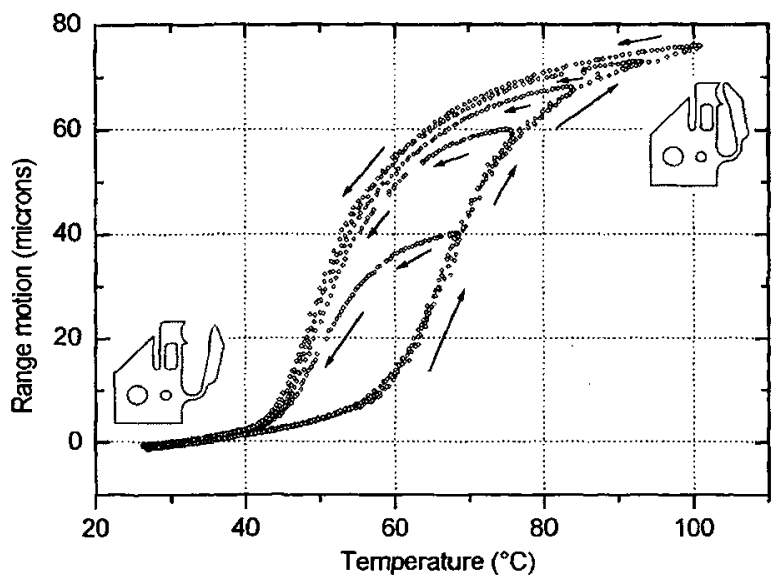

Figure 2: Successive partial cycles in the TWSME cycle induced by contrained thermal cycling

No SMART-effect (Step-wise Martensite to Austenite Reversible Transformation) as described by G. Airoldi et al. [6] have been observed.

The cycle shown on figure 2 , will be later considered as the "stable free cycle $»$. The question adressed in the next experiments was to investigate the behavior of the material when this latter is deformed in martensite in a position out of the stable free cycle. The results are shown on figure 3 . The gripper is mechanically deformed in the martensitic state in order to set to the shape in the closed position (A). When heating, surprisingly, the gripper does not keep the closed position but first opens until it reaches a critical point (B) where it goes back to the closed shape (C).

On cooling the gripper reopens following the stable cycle (C to D). If the device is then heated and cooled, the learned cycle is observed (D to C and the reverse). Therefore the effect of the «perturbation» has been cleared. This experiment has been reproduced for various levels of deformation in the martensitic state. The resuts are shown on figure 4 . Several interesting aspects can be pointed out:

- When deforms out of the stable cycle, the motion starts at a temperature below the martensite to austenite transformation temperature (Mf)

- The range motion is affected by the magnitude of the initial defor-

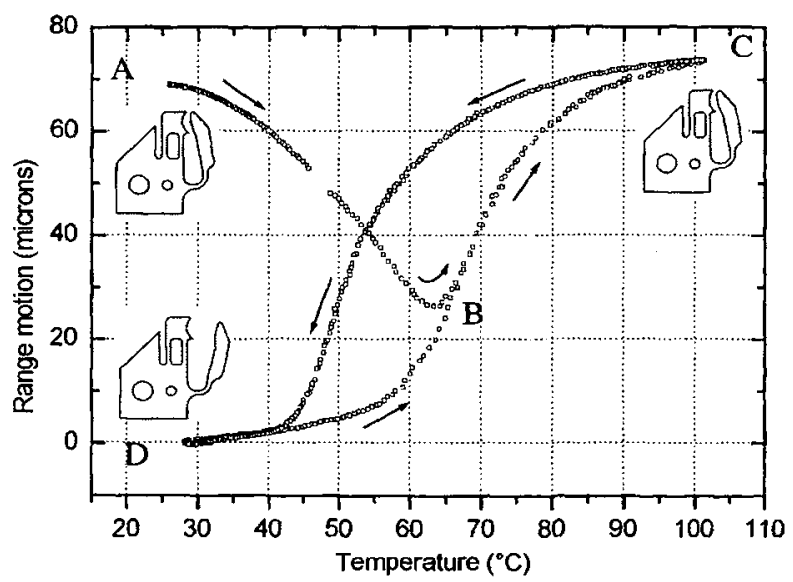

Figure 3: Behavior when starting from a position out of the stable free cycle. The applied sequence is A-B-C-D-C-D.

mation. As shown on figure $4 a$, there is a relationship with the deformation level and the loss of range motion. 


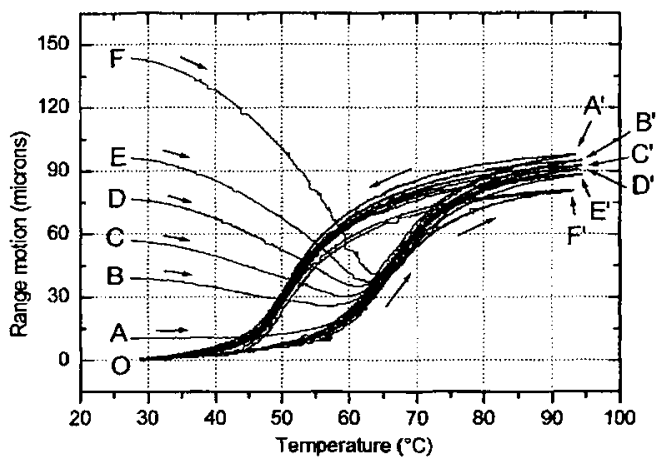

(a)

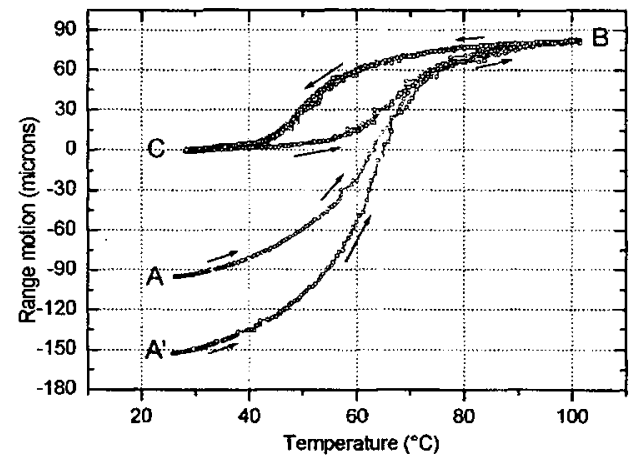

(b)

Figure 4: Device behaviour for different deformation levels out of the TWSME stable cycle. The curves on the left are corresponding to starts in the «closed direction* (sequences are $x-x^{\prime}-0-x^{\prime}-O$ where $\mathrm{x}=\mathrm{A}$..F) while the curves on the right are related to starts in kover-opened positions» (sequences $\mathrm{x}-\mathrm{B}-\mathrm{C}$ B-C where $\left.x=A, A^{\prime}\right)$.

- In the case of initial deformed position starting from the closed position, there is a minimal deformation to observe a direction change. For instance, starting from point $A$ on figure 4 does not lead to a direction change.

- If the gripper is placed in a "over-opened" initial state, the same phenomenon is observed. The transformation starts immediatly and curves seems to converge to the same zone on the stable cycle.

- Whatever the deformed position is, the position corresponding to a direction change upon heating when coming from a deformed position are almost in the same narrow range. Hence, the temperatures corresponding to a change in the motion direction are shown on figure 5 .

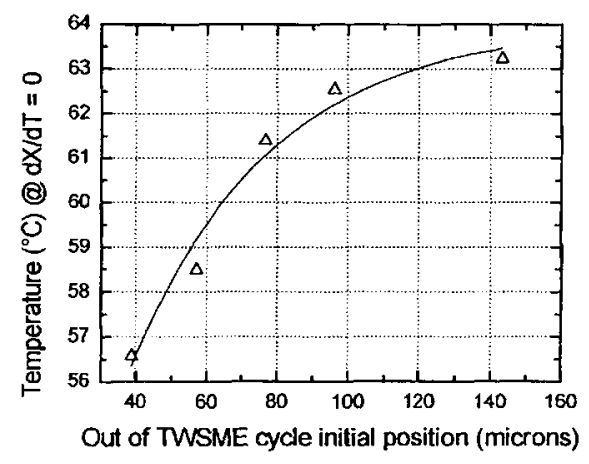

Figure 5: Approach to equilibrium temperatures as a function of the initial position.
These temperatures can be defined as the zeros on the position's derivates versus the temperature. Values are within eight degrees around $60^{\circ} \mathrm{C}$ and seems to follow a first order exponential law with the initial deformation. This temperature will be later mentionned as "the critical temperature in the approach to equilibrium" (Tc).

Let us call the curves presented on figure 4 , "approach to equilibrium" curves or $\mathrm{AE}$ curves where "the equilibrium" refers to the stable cycle obtained by the training process. As mentionned before, in the results shown on figure 4 , the motion starts immediately. Thus, it is interesting to determine the origin of this phenomenon and to investigate the material's behaviour in these approach to equilibrium curves. Hence, the gripper was initially set in the closed position and submitted to partial thermal cycles with a progressive increase of the magnitude. Results are shown on figure 6 . 


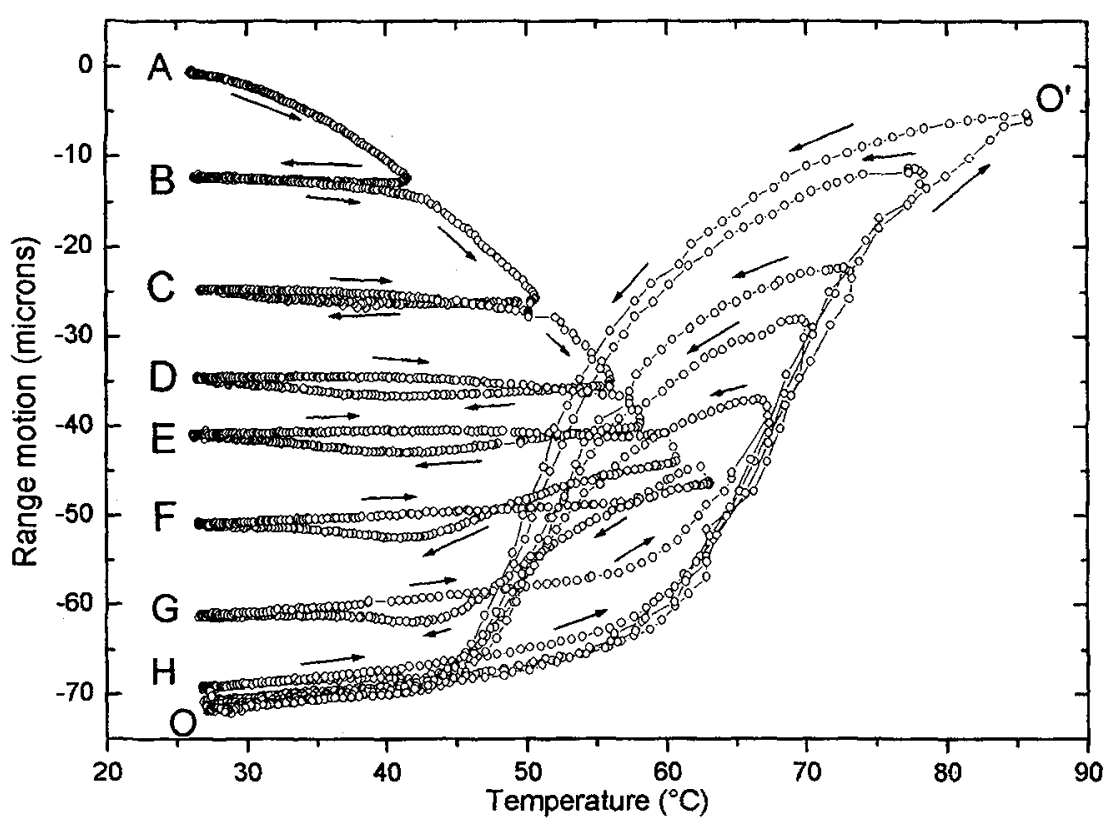

Figure 6: Sub-loops in an approach-to-equilibrium curve. The experiment chronology starts in $A$ then follows by the subloops $B, C, D, E, F, G, H$ until it reaches the equilibrium stable cycle going by subloops from $O$ to $O^{\prime}$.

The experiment starts in A corresponding to "the closed shape". Then, the device is heated up to a given temperature and then cooled down. This cycle is repeated several times with a progressive increase of the temperature magnitude thus producing the sub-loops ( $\mathrm{B}$ to $\mathrm{O}$ and $\mathrm{O}$ to $\left.O^{\prime}\right)$. In the first cycle (B) when the temperature decreases, the position remains stable on cooling. The position changes once again when the temperature reaches the point where the cycle was interrupted. Increasing the temperature at a level higher than the beginning of the stable cycle (Mf) leads to the appearance of a small hysteresis (sub-loop C). The hysteresis grows as the reached-temperature level increases with the cycles. The hysteresis in these sub-loops (for instance in sub-loop D) has an opposite sign compared to hysteresis observed in the stable cycle sub-loops.

\section{DISCUSSION}

The first thing that needs to be said is that these experiments deal with the approach to equilirium: a stable free cycle is obtained by the training process and then disordered by changing the initial condition. The stable free cycle is a consequence of micro-stresses induced by defects resulting from the training process. The disturbance is introduced by deforming the material in martensite either in the closed position direction or in the opposite one. It should be noted that this disturbance is mechanically prepared and thus can not spontaneously be reached by the material simply by changing the temperature.

If the temperature is increased from the ambiant temperature, these "prepared states" are not stable and disappear after a complete thermal cycle. Whatever the deformation direction was, the 
approach to equibilibrium curve seems to converge to a same area in the stable free cycle. In addition, the more important is the distance between the "perturbated" initial position and the "stable" initial position, the higher the convergence rate will be. This is clearly shown on figure 4. A first possible interpretation is the following: when disturbing the stable free cycle by modifying the initial condition, a two-step transformation occurs. In the approach to equilibrium (AE) curve, a martensite-to-martensite transformation is first observed. This transformation might be to a thermal-induced reorientation of martensitic variants in connection with the internal stress distribution created by the training process. This first phenomenom starts as the temperature is increased. It can be noted (see figure 6) that if the approach to equilibrium curve is stopped before a given temperature, which seems to correspond to the Mf temperature, no hysteresis is observed. When a given temperature higher than Mf is reached, some of the martensitic variants transform to austenite. This might explain why an hysteresis appears when the AE curve is stopped at a temperature higher than the $\mathrm{Mf}$ temperature. In addition, as the AE curve is getting closer to the stable cycle, this hysteresis increases. The peculiar shape of the hysteresis in this sub-loops - a motion first in the direction of the initial stable point on the stable cycle followed by a short reverse motion (subloops $\mathrm{F}$ and $\mathrm{G}$ on figure 6 , for instance) - might be due to a local minimum of energy corresponding to a given orientation of variants and leading to a metastable equilibrium. If the temperature cycles on the $\mathrm{AE}$ curve are further increased in magnitude, the effects of the disturbance are progressively wiped out.

As it can be seen on figure 4 , the initial positions have an effect on the magnitude of the TWSME cycle: the more important the perturbation is, the higher the decrease in magnitude will be. This might be explained by additionnal defects introduced by the deformation resulting in a loss of range motion.

\section{CONCLUSION}

These observations show that the TWSME is rather stable in deformation and can withstand disturbances in the "learned" cycle. When a perturbation is introduced, a two-step transformation occurs. This transformation may be a consequence of a competition between two phenomenon: a thermal induced reorientation of martensite variants and the martensite-to-austenite transformation. Future works will be done to further analyse this phenomenon in connection with the micro-structure of the trained material.

\section{Aknowledgements}

The authors like to thanks Prof Jan Van Humbeeck from the Katholieke Universiteit Leuven and Prof Max-Olivier Hongler from EPFL for the fruitful discussions.

\section{References}

1. J. Perkins, D. Hodgson in Engineering Aspects of Shape Memory Alloys, Edited by T. Duerig, A. Pelton, D. Stöeckel (Butterworth-Herneimann).

2. J. Van Humbeeck, R. Stalmans in Shape Memory Materials, Edited by K. Otsuka and C.M. Wayman. (Cambridge University Press, 1998).

3. Y. Bellouard, R. Clavel, J.-E. Bidaux, R. Gotthardt, T. Sidler, Journal de Physique (France) IV - 7 (1997), C5-603.

4. Y. Bellouard, T. Lehnert, J.-E. Bidaux, T. Sidler, R. Clavel, R. Gotthardt, Materials Sciences and Engineering A273-275 (1999), pp. 795-798.

5. A. Sulzmann, J. Jacot, in Proceeding of SPIE vol 3202, Microrobotics and Microsystem Fabrication, 16-17 Oct., Pittsburgh, USA (1997), edited by A. Sulzmann, pp. 42-51.

6. G. Airoldi, A. Corsi, G. Riva, Materials Science and Engineering A241 (1998) pp. 233-240. 